\title{
Prevalence of HIV among newly diagnosed tuberculosis patients in Erbil Governorate, Iraq
}

\author{
Zakarea Abdullah Yaseen Al-khayat ${ }^{1 \oplus *}$, Nabaz Fisal Shakir Agha ${ }^{2 \oplus}$, Pshtiwan Dhahir Majeed ${ }^{3}$, \\ Kawthar Ibrahim Fatah Alharmni ${ }^{4 \oplus}$, Derin Nabaz Fisal Agha ${ }^{5}$
}

\author{
'Department of Microbiology, College of Medicine, Hawler Medical University, Erbil, Iraq. \\ ${ }^{2}$ Department of Anesthesia, Erbil polytechnic university, Erbil Medical Technical Institute, Iraq. \\ ${ }^{3}$ Department of Nursing, Erbil polytechnic university, Erbil Medical Technical Institute, Iraq. \\ ${ }^{4}$ Department of Anatomy \& Histology, College of Medicine, Hawler Medical University, Erbil, Iraq. \\ 5. Department of Pharmacology, Erbil polytechnic university, Erbil Medical Technical Institute, Iraq. \\ *Correspondence to: Zakarea Abdullah Yaseen Al-khayat (E-mail: dr_zakarea@yahoo.co.uk) \\ (Submitted: 03 January 2021 - Revised version received: 22 January 2021 - Accepted: 06 February 2021 - Published online: 26 April 2021)
}

\begin{abstract}
Objective This study was accomplished with a purpose to determine the sociodemographic profile and the prevalence of HIV among tuberculosis (TB) patients.

Methods This prospective study was carried out in the Department of Microbiology at the Chest and Respiratory Disease Specialized Centre in Erbil City (In collaboration with the Specialist physician) through a period from January 2017 to December 2019. New TB patients were interviewed on a predesigned questionnaire. Collected samples were processed in a special laboratory in TB center. The samples were subjected to microscopy with Ziehl-Neelsen staining and inoculated on solid medium; the third sputum sample was tested directly by GeneXpert test. HIV testing was done using screening test and if the screening result was positive, the diagnosis was confirmed by Western Blot.

Results A total of 397 approved new TB patients underwent HIV testing. Among them, 41 cases (10.3\%) were found to be positive on ELISA screening, and subsequently they were all confirmed by the Western Blot test. The highest prevalence of HIV positivity according to gender, age range, and occupation, were as follows: male (29; 70.7\%), 30-42 years (21; 51.2\%), laborers (13; 31.7\%) respectively. The male to female ration is 2.7 statistically, the differences of distribution of the HIV positivity concerning the above-mentioned demography were as follows: gender: significant $(P \leq 0.05)$, age range: not significant, occupation: not significant. The highest prevalence of HIV positivity was among pulmonary TB $(25 ; 61 \%)$. Rifampicin resistant prevalence was higher among HIV positive in comparison to HIV negative TB case (23; $56.1 \%)(134 ; 37.4 \%)$, respectively. Statistically, the differences of distribution of the HIV positivity in relation to both TB pattern \& rifampicin monodrug resistant were significant $(P \leq 0.05)$.

Conclusions The prevalence of HIV infection in TB patients in current study was 10.3\%. If HIV testing was done for all TB patients, then routine reporting of HIV status for all TB patients would provide even better information which may provide a base to future planning.

Keywords HIV prevalence, Tuberculosis, Erbil, Sociodemographic determinants, Rifampicin resistance.
\end{abstract}

\section{Introduction}

Tuberculosis $(\mathrm{TB})$ is a paramount reason of morbidity and is amongst the peak 10 causes of death universally, standing over HIV as a sole infectious cause of death. ${ }^{1}$ Based on speculation from 2017, there were 10 million TB cases and 1.6 million TB-linked deaths worldwide, despite of a $2 \%$ incline in incidence proportion per annum in recent years. ${ }^{1}$ According to the World Health Organization (WHO), in 2019, 770,000 persons had passed away from HIV. An estimated third of these deaths were pertained to TB. ${ }^{2}$ HIV and TB are well known as a syndemic which is clarified as "the concourse of two or more diseases that perform in an interactive pattern to aggrandize the encumbrance of disease". ${ }^{3}$

Al-Salihy ${ }^{4}$ concluded that Iraq had been classified as a low-prevalence HIV epidemic, with a low number of formally reported cases $(0.1 \%$ of the total population). The first case was announced in 1986 among hemophilic patients who had received contaminated blood products. Furthermore, the cumulative number of HIV/AIDS registered cases from 1986 up to 2011 was 306. Among the registered cases, 85\% were male and the most common mode of transmission (66\%) was via imported blood products, $17 \%$ by heterosexual route, and $5 \%$ by vertical transmission from infected mother. No cases of transmission due to homosexual or drug addicts were reported. Since 2003, the transmission mode shifted towards the heterosexual route, as the government adopted strict measures to ensure blood safety.

Today, TB continues to be the main infectious reason of death in people with HIV, who are probably 15-22 times more likely to be infected with TB than people without. ${ }^{5}$ Presently, the Center for Disease Control and Prevention (CDC) advises that all patients with active or latent TB be checked for HIV because HIV is a quite-recognized stimulator of hidden TB. ${ }^{6}$ Nearly one-quarter of the universal inhabitance is appraised to be latently infected with Mycobacterium tuberculosis (Mtb) also referred to as latent tuberculosis (LTBI). ${ }^{7}$ A person with LTBI has an Mtb infection, but the bacteria continues to be recumbent and ineffectual within the host's body with no clinical markers. ${ }^{8}$ In HIV negative patients, a latent TB infection has a $10 \%$ chance of proceeding into an active infection throughout a lifetime. However, this jeopardy is considerably boosted in HIV positive patients and it progressively magnifies as immune function descends. ${ }^{9}$ Individuals with HIV and $\mathrm{TB}$ also have the potency to impact the health of HIV negative individuals because the brisk of LTBI makes Mtb highly infectious. ${ }^{10}$ Awareness about the predominance of HIV-positive infection in active TB patients is mandatory, as 
it is increasingly being realized that such facts would motivate arranging and may also be pivotal for setting the convenient treatment regime. ${ }^{11}$ The significance of HIV monitoring in TB patients is increasingly being recognized as the HIV epidemic keeps on to uphold the global TB epidemic. ${ }^{10}$ In numerous territories, the HIV dispersal in TB patients is a critical signal of the propagation of HIV into the overall inhabitance. Information about the HIV prevalence in TB patients is indispensable for supplying overall HIV/AIDS attention and assistance, encompassing antiretroviral therapy (ART) to HIV-positive patients. ${ }^{11,12}$

Thus, the objectives of this study were to explore the feasibility of HIV screening among TB patients registered for treatment in a selected TB treatment center in Erbil governorate and to depict the prevalence of HIV amongst TB patients in the research district. In addition, the study also describes various clinical presentations among these co-infected cases.

\section{Materials and methods}

\section{Study protocol}

This prospective study was carried out in the Department of Microbiology at the Chest and Respiratory Disease Specialized Centre in Erbil City (In collaboration with the Specialist physician) through a period from January 2017 to December 2019. This study was achieved with the cooperation of Prevention Health Department, Erbil Medical Technical Institute, Erbil Polytechnic University, with Departments of: Microbiology, Anatomy \& Histology, College of Medicine, Hawler Medical University, Erbil, Iraq.

\section{Moral considerations}

This study was confirmed by: the Ethics Committee of Hawler Medical University, Erbil; the Committee of Erbil Medical Technical Institute, Erbil Polytechnic University, Iraq; Health Directorate of Erbil. Acquainted endorsement was possessed from each patient. The patients were aware of study's goals and they could regress thereof if they wished so to do.

\section{Study population}

In the current study, all newly approved cases of TB (18 years of age and above) who were inhabitants of Erbil governorate were embroiled during the study period. All TB patients who conferred agreement for HIV testing (after pretest counseling) in the in- and outpatient wards of the Department of Medicine were involved in the study. Using a structured, pre-tested questionnaire including all personal information, such as age, gender, socioeconomic background, education level, profession, sexual preferences and promiscuity, history of past surgery, or blood transfusion were collected by patient interview. Patients receiving or who received anti-TB treatment in the previous month and non-consenting patients were excluded from this study. In addition, cases on ART who were attending the hospital for follow-up were also excluded.

\section{Sample and diagnostic procedures}

Suspected TB patients for collecting specimens were allocated in this study on the basis of presenting symptoms and chest radiography findings. Pulmonary specimens were taken from the sputum. Patients meeting the clinical eligibility criteria were asked to provide three sputum specimens: two spot samples and one obtained in the morning. Extra-pulmonary specimens were taken from pleural fluid, lymph node biopsy, gastrointestinal, cerebrospinal fluid, skin, and genitourinary.

\section{AFB and culture}

Collected samples were processed in a special laboratory in TB center, then two of the three samples were randomly selected and processed with $\mathrm{N}$-acetylcysteine and sodium hydroxide followed by centrifugation. ${ }^{13}$ The samples were resuspended in $1.5 \mathrm{~mL}$ of sample buffer and underwent microscopy analysis with Ziehl-Neelsen staining and cultured on solid medium (Löwenstein-Jensen, bioMerieux, France). The third sputum sample was tested directly by GeneXpert test. Solid cultures were considered negative after 42 days of incubation without isolation of any Mycobacteria. ${ }^{14}$

Non-respiratory specimens from closed and normally sterile sites were not decontaminated prior to smear preparation and culture but were concentrated by centrifugation at $3,000 \mathrm{~g}$ for $20 \mathrm{~min}$. Processed specimens from non-sterile sites and centrifuged specimens from sterile sites were directly cultured..$^{13,14}$

\section{GeneXpert assay}

The GeneXpert assay was used as previously used. ${ }^{15}$ Briefly, the provided buffer was added at a 3:1 ratio to clinical samples. The tubes were mixed manually twice in $15-\mathrm{min}$ period at room temperature before $2 \mathrm{~mL}$ of the inactivated material was transferred to the test cartridge. The cartridge was then inserted into the test platform, and the hand on work ended. Then, the machine automatically filtered, washed, and ultrasonically lyzed to release DNA. Real-time PCR amplification and detection were performed in an integrated reaction tube. Primers used for this assay were forward: (CGTGGAGGCGATCACACCGCAGAC) and reverse: (AGCTCCAGCCCGGCACGCTCACGT) (Applied Biosystems). The results were finally read after $1 \mathrm{~h} 45 \mathrm{~min}$, in which fluorescent signal was measured automatically. Negative or positive and defined susceptible or resistant to rifampin depending on the detection of mutations in $r p o B$ gene (MTBRIF Instructions). The patient confirmed to be infected with TB disease when: bacteriologically or microscopically confirmed pulmonary TB (PTB), i.e., positive culture and/or at least one sputum sample with a positive acid-fast bacillus (AFB) test; AFB positive smear which was a Fluorochromestained smear with at least one acid fast bacilli in 40 fields (or Xpert MTB/RIF positive). ${ }^{7,8}$

\section{HIV test}

HIV testing was done using KHB (Shanghi Kehua Bioengineering, Ltd, Shanghai, China) as a screening test. If the screening result was positive, the diagnosis was confirmed by Western Blot test (HIVBLOT 2.2, Genelabs Diagnostics, Singapore).

\section{Statistical analysis}

The data analysis was performed using descriptive statistics, including frequency, and frequency percentage. Comparisons were made using chi $^{2}$ test by using standard equations. The results were announced with $\mathrm{p} \leq 0.05$ or $\mathrm{p} \leq 0.01$ as the acceptable level of significance. 


\section{Results}

A total of $1436 \mathrm{~TB}$ patients were registered for treatment during the study period. Of the total TB patients, 397 had met the present study criteria mentioned above. The total 397 approved TB patients underwent HIV testing. Among them, 41 cases $(10.3 \%)$ were found to be positive on ELISA screening, and subsequently they were all confirmed by the Western Blot test. The remaining $356 \mathrm{~TB}$ cases were HIV negative.

Table 1 delineates the prevalence of HIV infection among new tuberculosis patients according to age, gender and occupation.

The highest prevalence of HIV positivity according to gender, age range and occupation, were as follows: male (29; $70.7 \%), 30-42$ years $(21 ; 51.2 \%)$, laborers $(13 ; 31.7 \%)$ respectively. The male to female ration is 2.7 . Statistically, the differences of distribution of the HIV positivity concerning the above-mentioned demography were as follows: gender: significant $(P \leq 0.05)$, age range: non-significant, occupation: non-significant

Table 2 illustrates TB pattern i.e.: pulmonary and extra pulmonary, and the rifampicin drug resistant among HIV seropositive and seronegative cases. The highest prevalence of HIV positivity was among pulmonary TB (25;61\%). Rifampicin resistant prevalence was higher among HIV positive in comparison to HIV negative TB case (23; 56.1\%) (134;

Table 1. Prevalence of HIV infection among new tuberculosis patients in relation to age, gender, occupation.

\begin{tabular}{|c|c|c|c|c|c|}
\hline Parameters & $\mathbf{N}$ & HIV+ & (\%) & HIV- & (\%) \\
\hline \multicolumn{6}{|l|}{ Gender } \\
\hline Male & 223 & 29 & (70.7) & 194 & (54.5) \\
\hline Female & 174 & 12 & (29.3) & 162 & $(45.5)$ \\
\hline \multirow[t]{2}{*}{ Total } & 397 & 41 & $(100)$ & 356 & (100) \\
\hline & \multicolumn{4}{|c|}{$\begin{array}{c}\chi^{2}=3.9373 \mathrm{df}=1, \\
\text { Significant }(P \leq 0.05)\end{array}$} & \\
\hline \multicolumn{6}{|l|}{ Age } \\
\hline $18-30$ & 116 & 11 & $(26.8)$ & 105 & (29.5) \\
\hline $30-42$ & 177 & 21 & $(51.2)$ & 156 & (43.8) \\
\hline$>42$ & 104 & 9 & (22) & 95 & (26.7) \\
\hline Total & 397 & 41 & $(100)$ & 356 & (100) \\
\hline \multicolumn{6}{|c|}{$\mathrm{df}=2 \mathrm{NS}$} \\
\hline \multicolumn{6}{|l|}{ Occupation } \\
\hline Farmer & 71 & 6 & (14.6) & 65 & (18.3) \\
\hline Service & 83 & 7 & (17.1) & 76 & $\begin{array}{l}(21.3) \\
\text { (clerk/ } \\
\text { tailor) }\end{array}$ \\
\hline Drivers & 79 & 10 & $(24.4)$ & 69 & (19.4) \\
\hline Housewives & 66 & 5 & $(12.2)$ & 61 & $(17.1)$ \\
\hline Laborers & 98 & 13 & (31.7) & 85 & (23.9) \\
\hline Total & 397 & 41 & (100) & 356 & $(100)$ \\
\hline \multicolumn{6}{|c|}{$\mathrm{df}=4 \mathrm{NS}$} \\
\hline
\end{tabular}

Table 2. Tuberculosis pattern and Rifampicin status among HIV seropositive \& seronegative patients.

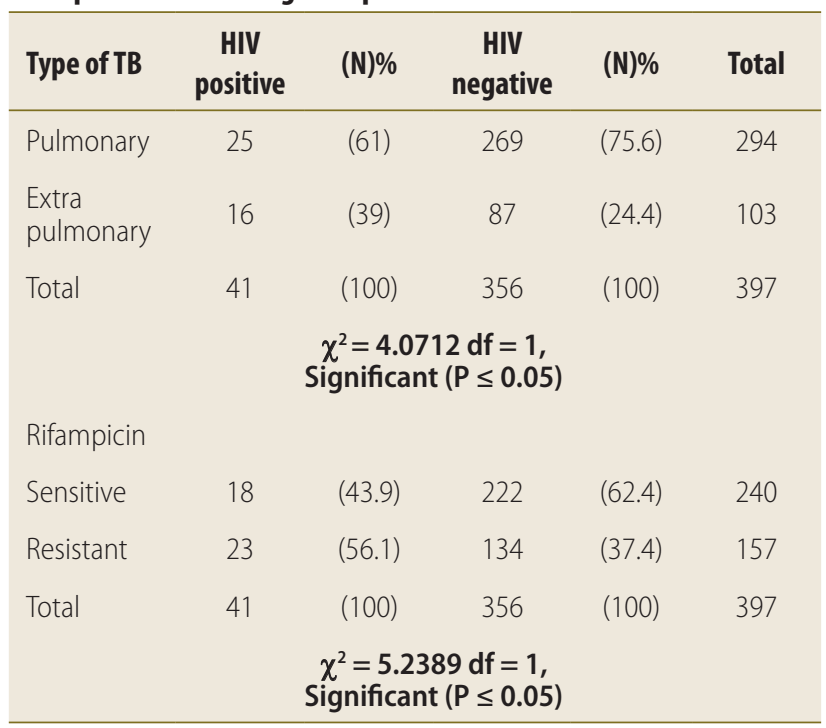

37.4\%) respectively. Statistically, the differences of distribution of the HIV positivity in relation to both TB pattern and rifampicin monodrug resistant were significant $(p \leq 0.05)$.

\section{Discussion}

To the best of our knowledge, no published data are available on the prevalence of HIV among newly diagnosed TB patients in Erbil governorate, hence this study can be considered the first study of such quality to deal with and investigate the prevalence, correlated risk factors, and impact of such an infection among these patients. According to the CDC recommendation, HIV screening to be accomplished for all TB patients comprising persons with $\mathrm{TB}$ disease, latent $\mathrm{TB}$ disease, and persons suspected of having TB and contact to TB patients. Thus, the current study was achieved to define the prevalence of HIV among diagnosed TB patients in Erbil. This study expounds that HIV seroprevalence among TB patients attending the Chest and Respiratory Disease Specialized Centre in Erbil City in Erbil in 2017-2019 was 10.3\%. Prevalence rates of HIV TB patients vary internationally. ${ }^{16}$ The present prevalence of HIV among TB patients was found to be less than $12 \%$, WHO's estimate of HIV among the world TB patients. ${ }^{17}$ The prevalence of TB/ HIV co-infection in India, ${ }^{18}$ and Ethiopia ${ }^{19}$ were $18.9 \%$ and $28.6 \%$, respectively, and were higher than our rates.

In a study carried by van der Werf et al. (20), the highest co-infection rates were recorded from Latvia (19.5\%), Malta (17.1\%), Portugal (14.7\%), and Estonia (10.1\%). Gao et $\mathrm{al}^{21}$ performed a systematic review and meta-analysis to determine the prevalence of TB/HIV co-infection by recognizing 47 studies comprising 272,466 persons except China. Their prime findings were that, a high prevalence of TB/HIV co-infection and they deduced that TB/HIV co-infection dispersal was higher among TB patients than among HIV/AIDS patients, but which were not significantly different.

Studies from Sub-Saharan Africa had enrolled those HIV seroprevalence rates of $50-70 \%$ in patients with tuberculosis. ${ }^{16}$

Hospital-based HIV seroprevalence studies amongst TB patients from various territories of India had exhibited 
a considerable divergences in the prevalence which varied from $0.4 \%$ to $28.1 \%$ have been reported. ${ }^{22}$ Studies performed in various provinces of Brazil had manifested that the prevalence of HIV infection in individuals with tuberculosis varies immensely, i.e., from $0.8 \%$ to $30 \%$, pertaining to the location studied and the technique applied. ${ }^{23}$ Furthermore, a research by Thanh et $\mathrm{al}^{24}$ determined fundamental geographical distinctions in HIV prevalence among TB patients.

These enormous diversities in the co-infection rates of TB/ HIV across the planet, as declared, can partially be attributed to: under-recording, diagnostic procedures used, discrepancies in TB diagnosis, epidemiology of TB in different nations, and study methodology utilized..$^{20,23,24}$

Table 2 explicates the prevalence of HIV seropositivity in regarding to gender, age, and occupational status.

Like other studies in different developing countries, ${ }^{17,18,25}$ the present study declared more prevalence of HIV co-infection amongst male TB patients.

Excepting a few nations in Africa (Sub-Saharan Africa), the prevalence of co-infection has been proclaimed to be higher among males than females. ${ }^{25-28}$ The prevalence of HIV is known to be higher among women than men in sub-Saharan Africa as a consequence of several reactions to sociodemographic risk factors, sexual attitude, and HIV/AIDS knowledge. Also, women are renowned to have a higher predisposition to HIV infection and are usually subjected to sexual behaviors earlier than men at most due to economic circumstances. ${ }^{27,28}$

Sentinel observational study accomplished in Delhi by Jain et al. ${ }^{29}$ in 1997-98 proposed a higher jeopardy of HIV co-infection existing among males complaining from $\mathrm{TB}$ which is in harmony with the present study. In the current study, HIV/TB patients were found to have a male-to-female ratio of 2.4. Others have found that the male/female ratio of $\mathrm{HIV}$-associated TB in Africa was $0.83,{ }^{28}$ although in other territories such as the Western Pacific, that ratio was 3.1. ${ }^{32}$ The ration of the current study is akin to the ratio of 2.7 revealed in 16 WHO European countries in 2005 and signaled that both infectious diseases are more widespread in the male population. ${ }^{30,31}$

With regard to the gender structure, it had been presumed that women with $\mathrm{TB}$ are not announced owing to a numerous of sociocultural issues, or diversified affairs affined with access in the health-care system and health services. ${ }^{16}$ Therefore, in spite of the biological elucidations, there are sturdy evidences that corroborate such a liaison between female under proclamation rates in the context of specific cultural factors which perform an important role in developing and transitional societies. ${ }^{25}$ Studies have been suggested that higher rate of TB infection in men may be due to the more vulnerability of males to pulmonary TB, difficulty in diagnosing TB in women, women with pulmonary $\mathrm{TB}$ have different symptoms from men and may not test positive on microscopic examination of the sputum, or that TB lung lesions might not be as severe in women as in men, resulting in women not being accurately diagnosed. ${ }^{18,22,32}$

In spite of the non-significant differences, the current study illustrate that HIV sero-positivity was higher among TB patients of age range 30-42 years and in laborer: $21(51.2 \%)$; $13(31.7 \%)$, respectively.

Studies had deduced that seroprevalence rates were highest among males in the age group between 31 and 40 years. ${ }^{19,22,27}$
HIV infection among TB patients continue to be astonishing, as it has been declared that more than 1 in $6 \mathrm{~TB}$ patients aged 25-44 year infected with HIV. ${ }^{25}$

The TB/HIV encumbrance was also had been observed to be higher within the age group that is mostly affected by TB. ${ }^{28}$ A study notified that the greatest leverage of the embarkation in TB extent had been on people between 25 and 45 years of age, since this is the age group mainly affected by HIV. ${ }^{29}$ This age prevalence of HIV co-infection among TB patients likely point out the age-specific prevalence of HIV in the community. This may be related to patients' being in a sexually active age group in which both TB and HIV dominate most. ${ }^{29,33}$ The other possible elucidation for this may be their increased family, organizational, and societal responsibilities as people in this age group involve themselves in various peculiar and distinctive daily activities in order to win the socioeconomic tribulation which increases the frequency of their contact with other patients in their society. ${ }^{28,34}$

The aforementioned outcomes of this study support this fact as the majority of the TB-HIV co-infected patients were in the productive age group 31-40 years, mostly illiterate and lack of skills. All these factors in linking with unsafe sexual practice were found to be the major cause of HIV transmission. ${ }^{16,22,24}$

$\mathrm{TB}$ is a disease of destitution. It is excessively realized that poorer community, the greater the probability of being infected with TB. Insufficiency of basic health services, poor nourishment and inappropriate living circumstances, all participate in the propagation of $\mathrm{TB}$ and its influence upon the society. ${ }^{16,24,27}$

In India, a study had determined that $76 \%$ of $\mathrm{TB} / \mathrm{HIV}$ cases were in the age group of 21-40 years and also the occupational profile of their patients disclosed that a majority of them were farmers and laborers followed by transport drivers. ${ }^{18}$ Channa et al. ${ }^{16}$ announced that $36.8 \%$ of TB/HIV patients were working as manual laborers while Thanh et al. ${ }^{24}$ found majority (55.6\%) of patients were working as farmers. Manjareeka ${ }^{22}$ had detected seropositivity proportion was elevated among those who were jobless (40\%) followed by the laborers (35\%).

The percentage of the professions is thus noted to alter in various studies, hugely due to the divergences in the occupational style and the origin from where the patients were picked. ${ }^{22}$ Some studies pointed out a strong interconnection of seropositivity with socioeconomic factor, whilst other studies linked knowledge and educational level. ${ }^{22,24}$

The current study revealed that the highest prevalence of HIV positivity was among pulmonary TB $(25 ; 61 \%)$. This result is consistent with findings reported by Chandra et al, ${ }^{35}$ Kamath et $a{ }^{1}{ }^{18}$ and Olowe et $\mathrm{al}^{36}$ where higher seropositivity of HIV were detected in cases of pulmonary TB. In addition, a study by Jha et $\mathrm{al}^{37}$ from eastern Nepal showed that $71.4 \%$ of the TB/HIV co-infected cases were suffering more commonly from pulmonary $\mathrm{TB}$ than with extra-pulmonary $\mathrm{TB}$ (28.6\%). The pulmonary TB (85.7\%) was more common than extra-pulmonary TB (14.3\%) was also reported by the study done in Western Nepal. ${ }^{38}$ Contrasting results have been found in studies conducted by Dahiya et $\mathrm{al}^{39}{ }^{39} \mathrm{Mitku}$ et $\mathrm{al}^{25}$, and Kavya et $\mathrm{al}^{40}$ with higher incidence of extra-pulmonary $\mathrm{TB}$ than pulmonary TB among HIV-TB co-infected patients.

Globally, the TB-associated HIV mortality in co-infected patients is three times higher than mortality among TB patients. ${ }^{3}$ There are a number of possible explanations that 
have been proposed for the increased mortality among co-infected patients. ${ }^{16,19}$ The location and the extent of TB are influenced by the degree of immunosuppression, often increasing the difficulty of diagnosis and hence delaying treatment initiation, which may result in higher mortality. ${ }^{9,1}$

With the scarcity of checking studies for EPTB and the deprivation of an appropriate diagnostic equipment, it is strenuous to estimate the actual spread of the disease or precisely evaluate the anatomical distribution of disease. ${ }^{20,22,24}$

Enormous retrospective explorations have been incapable to denote an appropriate matching amidst characteristics of EPTB because of the fickle appearance of the disease. ${ }^{16} \mathrm{~A}$ study in Brazil found similar non-specific symptoms in their patients with EPTB. ${ }^{23}$ The differential diagnosis for the existence of these fundamental symptoms is spacious, but stay as a substantial hint for the diagnosis of TB in a high TB/HIV load situation. Whilst symptoms and signs are beneficial in guidance; moreover, checking to confirm the location of disease, there is a shortage of data on distinguishing clinical findings that may be utilized to assist the diagnosis of TB over other pathologies at these sites. The clinical presentation of EPTB is protean, and establishing the diagnosis presents significant challenges in resource-limited settings. ${ }^{41,42}$

The deficiency of diagnostic devices take part to a diagnostic predicament, and future evolution in molecular established technology may ameliorate our capability to diagnose the disease adequately. The application of obtainable technologies demand better guide-based direction. Due to the reliance on non-microbiological diagnostic tests for EPTB, diagnostic certainty remains elusive. ${ }^{22,42}$

The complex relationship, however, between patient demographic characteristics, behavioral risk factors, clinical characteristics and comorbidities, and EPTB remains poorly understood. ${ }^{42}$

Studies revealed that newly diagnosed TB/HIV infected have greater risk of MDR-TB than those negative to HIV. ${ }^{43,44}$

While most studies in North America showed an association between HIV infection and MDR-TB, not a single study from Africa demonstrated such association, and results from other regions were conflicting. Individual studies varied widely in study design and sample size, and results were rarely adjusted for potential confounding. ${ }^{44}$ These restrictions and the noted diversity imped a comprehensive deductions considering the inclusive linking between HIV infection and MDR-TB. When stratified by type of MDR-TB, the analysis suggests that primary, but not acquired, MDR-TB is associated with HIV infection. ${ }^{45}$

Different biological mechanisms relating drug-resistant TB to HIV infection have been proposed. Drug malabsorption in HIV-infected patients, particularly rifampin and ethambutol, can produce drug resistance leading to treatment failure. ${ }^{46}$

The linkage between HIV infection and MDR-TB may be discomposed by risk factors such as injection drug use, imprisonment, socioeconomic status, alcohol use, and hospitalization which are shared between both. HIV-infected patients and MDR-TB patients are more likely to be hospitalized compared to those who are HIV negative.$^{47} \mathrm{HIV}$-infected patients may thus be more likely to be exposed to patients with drug-resistant isolates, and thus be infected or re-infected with a resistant isolate as the associations between MDRTB and HIV infection observed in many North American studies, which included in part patients involved in institutional outbreaks in New York City, support this possibility. ${ }^{46,47}$ It is of worthy to mention that Albujeer ${ }^{48}$ had concluded in their study in Iraq that preventing the spread of HIV in conservative communities such as Middle Eastern communities requires a comprehensive strategy that includes effective, continued health education and health promotion programs at both community and health professional levels.

\section{Conclusions}

The prevalence of HIV infection among TB patients in this study was $10.3 \%$. Greater focus of health intervention should be required on reproductive age group and in area in which high immigration and overcrowding present. Even with unavoidable restrictions, we can still determine that the HIV prevalence among TB patients was high. Involvement planes aiming sociodemographic and behavioral factors linking with higher risk of TB-HIV co-infection are imperatively needed for.

\section{Acknowledgments}

The authors are grateful to the management of Health Directorate of Erbil for their allowing us to conduct the study. We are also thankful to the senior staff and colleagues at the in the Department of Microbiology at the Chest and Respiratory Disease Specialized Centre in Erbil City for their cooperation and support.

\section{Financial support and sponsorship}

Nil.

\section{Conflicts of interest}

There are no conflicts of interest.

\section{References}

1. Harding E. WHO global progress report on tuberculosis elimination. The Lancet Resp Med. 2020 Jan 1:8(1):19.

2. World Health Organization. Global tuberculosis report 2020: executive summary.

3. Sharan R, Bucşan AN, Ganatra S, Paiardini M, Mohan M, Mehra S, Khader SA, Kaushal D. Chronic immune activation in TB/HIV co-infection. Trends Microbiol. 2020 Apr 22.

4. Al-Salihy SR, Enad OM. Knowledge and attitude of health care workers in Baquba Teaching Hospital toward HIV/AIDS infection. Iraqi J Public Health. 2017 Sep 20;1(2):42-6

5. Wong K, Nguyen J, Blair L, Banjanin M, Grewal B, Bowman S, Boyd H, Gerstner G, Cho HJ, Panfilov D, Tam CK. Pathogenesis of human

immunodeficiency virus-mycobacterium tuberculosis co-infection. J Clin Med. 2020 Nov:9(11):3575.

6. Melgar M, Nichols C, Cavanaugh JS, Kirking HL, Surie D, Date A, Ahmedov S, Maloney S, Fukunaga R, Offices'Tuberculosis CC, Advisors HI. Tuberculosis preventive treatment scale-up among antiretroviral therapy patients-16 countries supported by the US President's Emergency Plan for AIDS Relief, 2017-2019. Morbid Mortal Week Rep. 2020 Mar 27;69(12):329.

7. Sterling TR, Njie G, Zenner D, Cohn DL, Reves R, Ahmed A, Menzies D, Horsburgh Jr CR, Crane CM, Burgos M, LoBue P. Guidelines for the treatment of latent tuberculosis infection: recommendations from the National Tuberculosis Controllers Association and CDC, 2020: 1196- 1206. 
8. Bares SH, Swindells S. Latent tuberculosis and HIV infection. Curr Infect Dis Rep. 2020 Jul;22:1-8.

9. Letang E, Ellis J, Naidoo K, Casas EC, Sánchez P, Hassan-Moosa R, Cresswell F, Miró JM, García-Basteiro AL. Tuberculosis-HIV co-infection: Progress and challenges after two decades of global antiretroviral treatment roll-out. Arch Bronconeumol. 2020 Jan 10.

10. Ganatra SR, Bucşan AN, Alvarez X, Kumar S, Chatterjee A, Quezada M, Fish A, Singh DK, Singh B, Sharan R, Lee TH. Antiretroviral therapy does not reduce tuberculosis reactivation in a tuberculosis-HIV coinfection model. J Clin Investig. 2020 Aug 24;130(10).

11. Canetti D, Riccardi N, Martini M, Villa S, Di Biagio A, Codecasa L, Castagna A, Barberis I, Gazzaniga V, Besozzi G. HIV and tuberculosis: The paradox of dual illnesses and the challenges of their fighting in the history. Tuberculosis. 2020 May 1;122:101921.

12. Maher D, Watt CJ, Williams BG, Raviglione M, Dye C. Tuberculosis deaths in countries with high HIV prevalence: what is their use as an indicator in tuberculosis programme monitoring and epidemiological surveillance?[Unresolved Issues]. Int J Tuberculosis Lung Dis. 2005 Feb 1;9(2):123-7

13. Steingart KR, Ng V, Henry M, Hopewell PC, Ramsay A, Cunningham J, Urbanczik R, Perkins MD, Aziz MA, Pai M. Sputum processing methods to improve the sensitivity of smear microscopy for tuberculosis: a systematic review. Lancet Infect Dis. 2006 Oct 1;6(10):664-74.

14. Steingart KR, Henry M, Ng V, Hopewell PC, Ramsay A, Cunningham J, Urbanczik R, Perkins M, Aziz MA, Pai M. Fluorescence versus conventional sputum smear microscopy for tuberculosis: a systematic review. The Lancet Infect Dis. 2006 Sep 1;6(9):570-81.

15. World Health Organization. Rapid implementation of the Xpert MTB/RIF diagnostic test: technical and operational 'How-to'; practical considerations. World Health Organization; 2011

16. Channa AA, Jameel N, Khalil R. Prevalence of human immunodeficiency virus infection among the diagnosed tuberculosis patients in Karachi, Pakistan. Int J Res Med Sci. 2016 Mar;4(3):789.

17. Pourakbari B, Mamishi S, Banar M, Keshtkar AA, Mahmoudi S. Prevalence of TB/HIV co-infection in Iran: a systematic review and meta-analysis. Ann Ig. 2019 Jul 1;31(4):333-48

18. Kamath R, Sharma V, Pattanshetty S, Hegde MB, Chandrasekaran V. HIV-TB coinfection: Clinico-epidemiological determinants at an antiretroviral therapy center in Southern India. Lung India Off Organ Ind Chest Soc. 2013 Oct;30(4):302.

19. Belay M, Bjune G, Abebe F. Prevalence of tuberculosis, HIV, and TB-HIV co-infection among pulmonary tuberculosis suspects in a predominantly pastoralist area, northeast Ethiopia. Global Health Action. 2015 Dec 1;8(1):27949.

20. van der Werf MJ, Ködmön C, Zucs P, Hollo V, Amato-Gauci AJ, Pharris A. Tuberculosis and HIV coinfection in Europe: looking at one reality from two angles. AIDS (London, England). 2016 Nov 28:30(18):2845.

21. Gao J, Zheng P, Fu H. Prevalence of TB/HIV co-infection in countries except China: a systematic review and meta-analysis. PLoS One. 2013 May 31;8(5):e64915

22. Manjareeka M, Nanda S. Prevalence of HIV infection among tuberculosis patients in Eastern India. J Infect Public Health. 2013 Oct 1;6(5):358-62.

23. e Silva HO, Gonçalves ML. Prevalence of HIV infection in tuberculosis patients treated at primary health care clinics in the city of Fortaleza, Brazil. J Bras Pneumol. 2012;38(3):382-5

24. Thanh DH, Sy DN, Linh ND, Hoan TM, Dien HT, Thuy TB, Hoa NP, Tung LB, Cobelens F. HIV infection among tuberculosis patients in Vietnam: prevalence and impact on tuberculosis notification rates. Int J Tuberculosis Lung Dis. 2010 Aug 1;14(8):986-93.

25. Abdallah TM, Siddig MF, Ali AA. Seroprevalence of HIV infection among tuberculosis patients in Kassala, eastern Sudan. J AIDS HIV Res. 2011 Aug 31;3(8):161-3.

26. Mitku AA, Dessie ZG, Muluneh EK, Workie DL. Prevalence and associated factors of TB/HIV co-infection among HIV Infected patients in Amhara region, Ethiopia. Afric Health Sci. 2016 Jul 1;16(2):588-95.

27. Pennap G, Makpa S, Ogbu S. Sero-prevalence of HIV infection among tuberculosis patients in a rural tuberculosis referral clinic in northern Nigeria. Pan African Med J 2010;5:22

28. Baluku JB, Anguzu G, Nassozi S, Babirye F, Namiiro S, Buyungo R, Sempiira M, Wasswa A, Mulwana R, Ntambi S, Worodria W. Prevalence of HIV infection and bacteriologically confirmed tuberculosis among individuals found at bars in Kampala slums, Uganda. Scient Rep. 2020 Aug 10;10(1):1-9.

29. Jain SK, Aggarwal JK, Rajpal S, Baveja U. Prevalence of HIV infection among tuberculosis patients in Delhi — A sentinel surveillance study. Ind J Tuberculosis. 2000;47:21-6.

30. Fiebig L, Kollan C, Hauer B, Gunsenheimer-Bartmeyer B, an der Heiden M, Hamouda O, Haas W. HIV-prevalence in tuberculosis patients in Germany, 2002-2009: an estimation based on HIV and tuberculosis surveillance data. PLoS One. 2012 Nov 7;7(11):e49111.

31. Tavares AM, Fronteira I, Couto I, Machado D, Viveiros M, Abecasis AB, Dias S. HIV and tuberculosis co-infection among migrants in Europe: a systematic review on the prevalence, incidence and mortality. PLoS One. 2017 Sep 28;12(9):e0185526.

32. Hiatt T, Nishikiori N. Epidemiology and control of tuberculosis in the Western Pacific Region: analysis of 2012 case notification data. Western Pacific Surveil Resp J:WPSAR. 2014 Jan;5(1):25

33. Jaiswal RK, Srivastav S and Mahajan H. Socio demographic profile of TB-HIV coinfected patients in Bundelkhand region,Uttar-Pradesh. Nat Jn Med Research 2012; 2(2):149-151.

34. Soyam VC, Das J, Rajeeva TC, Boro P, Kohli C. Prevalence and sociodemographic correlates of HIV among tuberculosis patients of DOTS centre in Delhi. Asian J Med Sci. 2016;7(1):53-8.

35. Chandra NM, Babu RA, Prasad DTS, Devulapalli M, Banu SSK, Avanthi B, et al. Epidemiological surveillance of tuberculosis among HIV/AIDS seropositive individuals attending ART center at a tertiary care teaching hospital. Int J Community Med Public Health 2017:4:2816-2824.

36. Olowe OA, Makanjuola OB, Adekami AS, Adefioye OJ. Epidemiological characteristics and clinical outcome in a population of TB patients in SouthWestern Nigeria. Eur J Microbiol Immunity 2017; 2: 127-132.

37. Jha N, Khanal B, Karki PP, Rijal S, Deo BK, Khadka DK, Malla P. TB/HIV coinfection status among the newly diagnosed TB patients: A Study from Eastern Nepal. SAARC J Tubercul Lung Dis HIV/AIDS. 2008;5(2):22-5.

38. Ghimire P, Dhungana GR, Bam DS, Rijal BP. Tuberculosis and HIV co-infection status in United Mission Hospital, Tansen, Western Nepal. SAARC J Tubercul Lung Dise HIV/AIDS. 2004;1:32-7.

39. Dahiya N, Bachani D, Das R, Rasania SK. Socio-demographic and clinical profile of HIV positive patients attending integrated counseling and testing center of a primary health centre in Delhi. SAARC J Tubercul Lung Dis HIV/ AIDS 2017; 15:22-26

40. Kavya S, Anuradha K, Venkatesha D. CD4 count evaluation in HIV-TB co infection before and after anti-tubercular treatment. Int J Res Med Sci. 2017;2(3):1031-4

41. Leeds IL, Magee MJ, Kurbatova EV, del Rio C, Blumberg HM, Leonard MK, Kraft CS. Site of extrapulmonary tuberculosis is associated with HIV infection. Clin Infect Dis. 2012 Jul 1:55(1):75-81.

42. Namme LH, Marie-Solange D, Hugo Bertrand MN, Elvis T, Achu JH, Christopher K. Extrapulmonary tuberculosis and HIV coinfection in patient treated for tuberculosis at the Douala General Hospital in Cameroon. Ann Trop Med Public Health 2013:6:100-4.

43. van den Hof S, Tursynbayeva A, Abildaev T, Adenov M, Pak S, Ismailov S. HIV and multidrug-resistant tuberculosis: overlapping risk factors. Eur Resp J. 2015 Feb 1;45(2):567-9.

44. Adetunji SO, Donbraye E, Ekong MJ. HIV infection among rifampicin resistant tuberculosis patients in Ibadan, Southwest Nigeria. World J. Med. Sci. 2018;15(4):139-43

45. Singh A, Prasad R, Balasubramanian V, Gupta N. Drug-resistant tuberculosis and HIV infection: current perspectives. HIV/AIDS (Auckland, NZ). 2020;12:9.

46. Saldanha N, Runwal K, Ghanekar C, Gaikwad S, Sane S, Pujari S. High prevalence of multi drug resistant tuberculosis in people living with HIV in Western India. BMC Infect Dis. 2019 Dec;19(1):1-6.

47. Baluku JB, Mugabe P, Mulwana R, Nassozi S, Katuramu R, Worodria W. High Prevalence of rifampicin resistance associated with rural residence and very low bacillary load among TB/HIV-coinfected patients at the national tuberculosis treatment center in Uganda. BioMed Res Int. 2020 Jul 25;2020

48. Albujeer AN, Shamshiri AR, Taher A. HIV/AIDS awareness among Iraqi medical and dental students. J Int Soc Prev Commun Dentistry. 2015 Sep:5(5):372 